\title{
Partial incubation-induced changes in concentrations of egg white antimicrobials do not influence trans-shell infection but affect hatchling phenotype
}

\author{
Jana Svobodová \\ Czech University of Life Sciences Prague \\ Jakub Kreisinger \\ Charles University \\ Veronika Gvoždíková Javůrková ( $\nabla$ veronika.javurkova@gmail.com ) \\ Institute of Vertebrate Biology
}

\section{Research Article}

Keywords: Anas platyrhychos, AMPs, Microbes, lysozyme, phenotype

Posted Date: March 23rd, 2021

DOI: https://doi.org/10.21203/rs.3.rs-337108/v1

License: (c) (i) This work is licensed under a Creative Commons Attribution 4.0 International License.

Read Full License 
1 Partial incubation-induced changes in concentrations of egg white antimicrobials do not

2 influence trans-shell infection but affect hatchling phenotype

3

4 Jana Svobodová ${ }^{1}$, Jakub Kreisinger ${ }^{2}$, Veronika Gvoždíková Javůrková ${ }^{3,4}$ *

5

6 1. Czech University of Life Sciences, Faculty of Environmental Sciences, Department of 7

2. Charles University, Faculty of Science, Department of Zoology, Viničná 7, 12844

3. Czech Academy of Sciences, Institute of Vertebrate Biology, Květná 8, 60365 Brno,

4. Czech University of Life Sciences, Faculty of Agrobiology, Food and Natural Resources, Department of Animal Science, Kamýcká 957, 16500 Prague 6, Czech Republic

* Corresponding author:

Veronika Gvoždíková Javůrková (ORCID ID: https://orcid.org/0000-0002-9914-0905)

Czech Academy of Sciences, Institute of Vertebrate Biology, Květná 8, 60365 Brno, Czech Republic 
Host-microbiome interactions during embryonal and early phase of life is critical point in microbiome formation and assemblage in neonates. In birds, transmission of microbes from the outer environment into the egg interior was found to shape embryo viability and hatchlings phenotype. Microbes' transmission may be modulated by egg white antimicrobial proteins (AMPs) whose concentration and antimicrobial action are temperature-modulated. As partial incubation and clutch covering with nest-lining feathers during pre-incubation period may both significantly alter temperature conditions acting on eggs, we experimentally investigated effects of these behavioural mechanisms on the concentrations of primary egg white AMPs - lysozyme and avidin using Mallard (Anas platyrhychos) eggs. Moreover, we studied in vivo if concentrations of egg white AMPs reduced probability and intensity of bacterial trans-shell infection and hatchlings phenotype. We found significantly higher egg white lysozyme concentration, while avidin concentration tended to be higher in partially incubated eggs. Clutch covering with nest-lining feathers had no effect on egg white AMPs concentrations. Neither probability nor intensity of bacterial trans-shell infection was associated with concentrations of egg white AMPs. Finally, increased egg white lysozyme was associated with decreased scaled body mass index of hatchlings. These outcomes demonstrate that incubation prior to clutch completion in precocial birds may modulate concentrations of particular egg white AMPs, yet without any effect on transmission of bacteria into the egg in vivo. Furthermore, increased egg white lysozyme may compromise body condition of hatchlings supporting growth-regulating role of lysozyme during embryogenesis in precocial birds. 
53 Microbiome interactions and assembly in neonates seems to be determining for prosperity and overall success of progeny ${ }^{1-4}$. The most recent concept suggests so called "nidobiome" as interconnected system shaping the microbial colonization of neonates and thus a new unit of host-microbiome interactions ${ }^{5}$. This concept integrates parents, nest and neonates per se as the main modifiers of microbiome assembly in progeny ${ }^{5}$. Based on this concept, eggshell microbiome and penetration of the eggshell microbes into the egg content (i.e. microbial transshell infection) could be considered as one of the critical sources of nidobiome.

Avian eggshell microbiome has been found to be diverse ${ }^{6,7}$ and primarily shaped by the nest material and environment and parents' skin and feathers ${ }^{8-11}$. Microorganisms have, however, also ability to enter avian egg interior, as was documented under both natural ${ }^{12}$ and experimental conditions ${ }^{13,14}$. Unlike broadly documented associations between eggshell microbiota and hatching success ${ }^{15,16}$, studies investigating proximate effects of penetrated microorganisms on avian embryo and hatchlings are scarce with only a few documenting suppressed embryo viability ${ }^{12,17-19}$ or decreased residual body weights of hatchlings ${ }^{13}$. Considering diversified eggshell microbiome and its ability to enter egg interior, numerous eggrelated and behavioural mechanisms protecting against uncontrolled proliferation of microbes outside and inside the eggs have been evolved in birds.

Eggshell pigmentation $^{20}$, eggshell microstructure characteristics ${ }^{21-23}$, cuticle nanostructuring ${ }^{24}$, 71 deposition of antimicrobial proteins into the eggshell structures ${ }^{25-27}$ and nest material ${ }^{8,10,28}$ have 72 been found to significantly shape eggshell microbiota. Nevertheless, mechanisms reducing 73 microbial trans-shell infection and proliferation of microbes inside the eggs are primarily linked 74 with egg incubation ${ }^{29,30}$ and the concentrations of abundant egg white proteins ${ }^{31,32}$, especially 75 those having antimicrobial potential ${ }^{30,33,34}$. 
Among the most investigated and abundant egg white antimicrobial proteins (AMPs) are lysozyme, ovotransferrin and avidin ${ }^{32,35}$. While lysozyme has strong bactericidal activity against both $\mathrm{G}+$ and $\mathrm{G}-$ bacteria $^{33,36}$, avidin and ovotransferrin are rather bacteriostatic ${ }^{33,37,38}$ due to their ability to reversibly bind biotin and iron thus making them unavailable for bacterial growth $^{39-41}$. Howerver, despite documented antibacterial action of egg white AMPs, egg white proteomic profile may be significantly changed during incubation ${ }^{42,43}$ leading to alterations in egg white antimicrobial potential ${ }^{29,30,44,45}$. For example, changes of egg white lysozyme and ovotransferrin concentrations were found to enhance proliferation of beneficial probiotic microorganism in egg white ${ }^{30}$. Therefore, while incubation may significnatly shift eggshell microbiota $^{6,45-48}$, its selective antimicrobial effect inside the egg is most probably inherent in mediation of changes in chemical and proteomic profile of the egg white. Furthermore, as evidence exists for physiological role of lysozyme and avidin on developing embryo resulting in alterations of hatchlings phenotypic traits ${ }^{49,50}$, incubation-mediated changes in egg white AMPs profile might also significantly shape hatchlings' phenotype. Despite these facts, experimenal evidence for the interactive effects of egg white AMPs profile and incubation under natural conditions are lacking.

Another behavioural mechanism with potential to reduce risk of microbial trans-shell infection is clutch covering with nest lining material during pre-incubation period. Recent studies revealed the role of nest material and nest-lining feathers on eggshell microbial assemblage most probably related to antimicrobial agents produced by microorganisms in preen gland $^{8,10,28}$. Excepting such direct antimicrobial action of nest-lining feathers, clutch covering protects exposed clutch against temperature fluctuations caused by ambient temperatures during pre-incubation period ${ }^{51,52}$. Although in our previous experimental study clutch covering with the nest lining had no effect on bacterial trans-shell infection ${ }^{13}$, it may affect temperature properties on exposed eggs and induced temperature-mediated changes in egg white AMPs 
114 other precocial birds ${ }^{49,50,57}$, we predicted ducklings' phenotype will be modified owing to 115 variability in egg white lysozyme and avidin concentrations.

\section{Material and Methods}

\section{Ethical statement} Republic.

concentrations. Nevertheless, proximate role of clutch covering with feather nest lining on egg white AMPs concentration has not yet been evaluated to date.

Partial incubation is behaviour preceding full incubation of complete clutch in many bird species ${ }^{53}$ with documented function to keep eggs dry ${ }^{54}$, modulate eggshell microbiota ${ }^{18,48}$, or have antipredator role ${ }^{55,56}$. Yet, if partial incubation may affect or stabilize antimicrobial properties of egg content is unknown. In this study, we experimentally tested if partial incubation and clutch covering with feather nest lining during pre-incubation period affect concentrations of two principal egg white AMPs - lysozyme and avidin using precocial mallard eggs exposed in natural breeding habitat. Furthermore, as in our previous study ${ }^{30}$ experimental increase of egg white AMPs concentrations enhanced in vitro antimicrobial activity of egg white against selected bacterial strains, we hypothesized that different concentrations of egg white AMPs will affect probability and intensity of bacterial trans-shell infection in vivo. Finally, due to documented phenotype modulating roles of egg white lysozyme and avidin in

All experiments and analyses were performed in accordance with relevant institutional guidelines and regulations. The field experiment was carried out under permission no. 162 (15/2/2006), issued by the Ministry of Environment, on behalf of the Government of the Czech Republic.

\section{Experimental procedures}


126 We applied experimental approach with freshly laid Mallard eggs $(n=160)$ obtained from a

127 commercial hatchery (Mokřiny Duck Farm, Třeboň Fisheries Ltd, Czech Republic) in June

128 2010. Experimental eggs were randomly selected over a single day, ensuring that they came

129 from different females. To compute egg volume (Rohwer 1988) eggs length and width were

130 measured with digital callipers (0.01 mm accuracy; Kinex, Prague, Czech Republic).

131 Subsequently, each egg was cleaned with $70 \%$ ethanol to eliminate the initial eggshell bacterial

132 assemblage, and placed into sterile portable boxes.

133 Four randomly selected mallard eggs were placed into each experimental nest

134 distributed in typical breeding habitat of Mallards (Dívčice, Czech Republic $\left(49^{\circ} 6^{\prime} \mathrm{N}, 14^{\circ} 18\right.$

E). Eggs in each nest were sorted based on a balanced $2 \times 2$ factorial design (see Fig. 1 in ${ }^{13}$

136 and for all experiment details); two eggs were covered with a mixture of feather nest-lining

137 collected from active mallard nests and two eggs remained uncovered. In addition, two eggs

138 (one covered and one uncovered) were incubated daily in an incubator (OvaEasy 190 Advance,

139 Brinsea Products Inc., Titusville, FL, USA) for periods that mimicked the pattern of partial

140 incubation observed for Mallards during the egg-laying period ${ }^{13,58}$ (see Table S1 in ${ }^{13}$, for

141 details). The two remaining eggs were not incubated. Incubated eggs were transferred from

142 experimental nests to the incubator and back each day in portable sterilized boxes. Total 143 exposure of incubated eggs was for $45 \mathrm{~h}$ at $37.6{ }^{\circ} \mathrm{C}$ with a relative humidity of $60 \%$. Eggs were 144 turned $180^{\circ}$ twice daily to maintain optimal egg hatchability ${ }^{59}$. All experimental procedures 145 were described in ${ }^{13}$, in detail.

147 Egg white sampling and assessment of egg hatchability

148 Egg white sampling procedures are identical with those described in $^{13}$. Particularly, all 149 experimental eggs were cleaned with $70 \% \mathrm{EtOH}$ and their shell was gently perforated with a $15022 \mathrm{G}(0.7 \times 40 \mathrm{~mm})$ sterile needle (Terumo ${ }^{\circledR}$, Germany) at the blunt end. Thereafter, $300 \mu \mathrm{L}$ 
151 of egg white removed with a $0.5-\mathrm{mL}$ sterile syringe (B Braun, Germany) and in sterile cryotubes

152 stored at $-20{ }^{\circ} \mathrm{C}$. Needle perforations in the eggshell were sealed using a gel-based adhesive

153 (Loctite-Super Attack, Henkel, USA). Based on previous studies ${ }^{60,61}$, such a procedure does not

154 significantly affect the hatchability of eggs. To assess eggs hatchability, the eggs were placed

155 back in the incubator with temperature and relative humidity at $37.6{ }^{\circ} \mathrm{C}$ and $60 \%$, respectively,

156 and relative humidity was increased to $80 \%$ during the egg-hatching period ${ }^{62}$. The weight $( \pm$

$1570.1 \mathrm{~g})$ and tarsus-length $( \pm 0.1 \mathrm{~mm})$ of each duckling was measured immediately after hatching.

158 An egg was assigned as fertile in the case of its successful hatching or in the presence of a

159 cicatricule or dead embryo inside an un-hatched egg ${ }^{63}$.

160

$161 \quad$ AMPs concentrations

162 Concentration of egg white lysozyme

163 Lysozyme concentration was measured using an agar well-diffusion assay ${ }^{64}$ with $50 \mathrm{mg}$ of

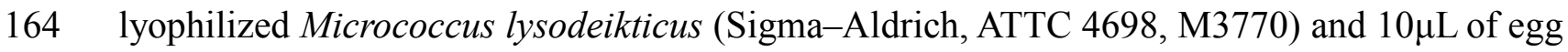

165 white samples that were transferred in duplicates into the holes on agar plates. Standard

166 solutions $(10 \mu \mathrm{L})$ of known concentrations $(20,15,7,4,2,0.5 \mathrm{mg} / \mathrm{mL})$ of lyophilized hen egg

167 white lysozyme (62971, Fluka) was added into the holes of each agar plate. The plates were

168 incubated for 24 hours at $21^{\circ} \mathrm{C}$ and $50-60 \%$ humidity. Photographs of plates with clearance

169 zones around the holes were analysed using ImageJ. Lysozyme concentrations $(\mathrm{mg} / \mathrm{mL})$ for

170 each egg white sample were interpolated from a calibration curve using GraphPad Prism

171 version 6.00 for Windows (GraphPad Software, San Diego California USA). Methods for

172 measuring of lysozyme concentration is described in detail in ${ }^{30}$.

173

174 Concentration of egg white avidin 
175 Avidin concentration $(\mu \mathrm{g} / \mathrm{mL})$ was based on a slightly modified version of the 96-well plate

176 method of ${ }^{65}$. We diluted each egg white sample 10-fold in carbonate-bicarbonate buffer (made

177 from Sigma-Aldrich C3041 capsules, following the manufacturer's instructions). We then

178 added $100 \mu \mathrm{L}$ of carbonate-bicarbonate buffer to each well (except the first, fifth and ninth well

179 in each row) along rows one to 11 of a Nunc MaxiSorp ${ }^{\circledR}$ flat-bottom 96-well plate. The wells

180 in the bottom row 12 contained $100 \mu \mathrm{L}$ of avidin standard solution $(2.5-0.002 \mu \mathrm{g} / \mathrm{mL}$; Sigma

181 Aldrich; A9275) diluted in carbonate-bicarbonate buffer. In order to ensure accurate pipetting

182 of undiluted and diluted egg white samples, we used GENO-DNA S pipette tips (CS960

183 9405120, Thermo Fisher Scientific), specially designed for viscous liquids. The 96-well plate

184 was then sealed with parafilm and incubated at $4{ }^{\circ} \mathrm{C}$ overnight. The next day, the content of the

185 wells was poured out and the plate rinsed three times by adding $200 \mu \mathrm{L}$ of $0.05 \%$ Tween

186 washing buffer (Tween 20/PBS) to each well and shaking for five minutes on an IKA KS 260

187 basic lab shaker. Non-specific protein sites were blocked by adding $200 \mu \mathrm{L}$ of blocking buffer

188 (1\% solution of bovine serum albumin (Sigma Aldrich) in PBS) to each well three times for 30

189 sec. Then, $100 \mu \mathrm{L}$ of a 1:4000 dilution of Superblock buffer (0.05\% Tween 20/blocking buffer)

190 with HRP (Invitrogen, Thermo Fisher Scientific) was added to each well and incubated at room

191 temperature for 25 minutes. The wells were then washed five times with $200 \mu \mathrm{L}$ of washing

192 buffer, followed by $30 \mathrm{sec}$. shaking on the lab shaker. We then added $100 \mu \mathrm{L}$ of TMB Substrate

193 blocking buffer (Sigma Aldrich) to each well and incubated the plate at room temperature for

19430 minutes. The reaction was stopped by adding $100 \mu \mathrm{L}$ of TMB Substrate Stop Reagent (Sigma

195 Aldrich) to each well and mixing it. Samples absorbances were measured at $450 \mathrm{~nm}$ using a

196 TECAN Infinite ${ }^{\circledR} 200$ PRO UV/Vis microplate reader (Tecan Group, Männedorf,

197 Switzerland). Each sample was analysed in duplicates. Avidin concentrations (considering four

198 egg white serial dilutions) were determined by interpolating from a standard curve for each 
plate using GraphPad Prism 5 Software (inter-assay and intra-assay coefficients of variability were $12.6 \%$ and $3.2 \%$, respectively).

Analysis of bacterial trans-shell infection

Bacterial genomic DNA was extracted from egg white using the EliGene MTB Isolation Kit

(Elisabeth Pharmacon, Brno, Czech Republic). Incidence and intensity of bacterial trans-shell infection (BTSI) were analysed using RT-PCR based entirely on the targeting of 16S rRNA using an RT-PCR LightCycler ${ }^{\circledR} 480$ system (Roche, Mannheim, Germany). The LightCycler 480 SYBR Green I Master (Roche) and the universal Eubacteria primer set, including forward primer Uni331 (5'-TCCTACGGGAGGCAGCAGT-3') and reverse primer Uni797 (5'GGACTACCAGGGTATCTAATCCTGTT-3'), were used for RT-PCR amplification ${ }^{66}$.. Serial 210 dilutions $\left(10^{1}\right.$ to $\left.10^{9}\right)$ of purified genomic Streptococcus bovis DNA from known number of 211 bacterial cells were used to construct calibration curves for quantification of BTSI intensity, expressed as number of bacterial cells per $1 \mathrm{~mL}$ of egg white. Details on conditions for DNA amplification are in detail described in $^{13}$.

\section{Statistics}

As AMPs measurements were highly repeatable (interclass correlation coefficient $=0.86$ for avidin and 0.95 for lysozyme), we used average avidin and lysozyme concentration values of each biological sample for all later analyses. Moreover, concentrations of avidin and lysozyme were not correlated (Spearman correlation, rho $=0.01, p=0.869$ ). We therefore built separate models predicting concentrations of these two AMPs. Eggs were clustered into quadruplets during the experimental phase of our study, which may affect probability of BTSI as shown in our previous study ${ }^{13}$. To account for this source of data non-independence, quadruplet's identities were included as random intercepts into all models, if not otherwise stated. Using 
volume is related to AMPs concentrations and if AMPs concentrations were affected by partial

226 incubation, clutch covering and interactions between these two variables. The effect of protein concentration along with effects of the above mentioned incubation treatments on incidence of BTSI and hatching success were analysed using logistic GLMMs (binomial error distribution, logit link). Next, using a subset of eggs with positive BTSI (i.e. number of bacterial cells 230 estimates per $1 \mathrm{ml}$ of albumen $>1$ ) and GLMMs with Gaussian error distribution, we asked if 231 the intensities of bacterial infection $\left(\log ^{10}\right.$ scaled) were predicted by concentrations of the two 232 AMPs. Finally, we searched for an association between AMPs concentrations and hatchling's phenotype. To do so, we used (i) body mass adjusted for the effect of egg volume (i.e. residuals

234 from a linear regression on body mass vs. egg volume) and (ii) scaled body mass index ${ }^{67}$ as 235 response variables. AMPs concentrations, along with the effect of partial incubation (that 236 considerably affects hatchling's phenotype ${ }^{13}$ ) were used as predictors. Effect of AMPs on 237 phenotype traits was modelled using linear regression since mixed models exhibited poor 238 convergence on this data subset. Moreover, there were only two quadruplets with more than a 239 single egg successfully hatched, suggesting negligible effect of data non-independence on the 240 outcomes of these analyses.

As data on avidin concentrations exhibited skewed distribution, we used its $\log ^{10}$ transformed values in all statistical calculations. Models were fitted using R software ${ }^{68}$ running 243 in Rstudio (version 1.1.453) ${ }^{69}$ and package $1 \mathrm{me} 4^{70}$. Backward elimination of non-significant 244 terms in the GLMM was used to select the best minimal adequate model (MAM), i.e. the most 245 parsimonious model with all effects significant ${ }^{71}$, first eliminating non-significant interactions 246 and subsequent non-significant main effects. The significance of a particular explanatory 247 variable was derived from the change in deviance between the model containing this term and 248 the reduced model assuming $\chi^{2}$ or $\mathrm{F}$ distribution of difference in deviances, with degrees of 249 freedom equal to the difference in degrees of freedom between the models with and without the 
term of question ${ }^{71}$.

\section{Results}

Effect of partial incubation and clutch covering with nest-lining feathers on egg white AMPs

There was no association between egg volume and lysozyme or avidin concentrations $(\Delta$ d.f. $=$ $1, \chi^{2}=0.05, p=0.816$ and $\chi^{2}=0.33, p=0.566$, respectively; Table 1$)$. Lysozyme concentrations increased in partially incubated eggs $\left(\Delta\right.$ d.f. $=1, \chi^{2}=25.72, p<0.001$; Table 1 and Figure 1$)$. Similar, yet nonsignificant trend was observed for avidin $\left(\Delta\right.$ d.f. $=1, \chi^{2}=3.28, p=0.070$; Table 1 and Figure 1). There was, however, no effect of clutch covering or the interaction between clutch covering and partial incubation on avidin and lysozyme concentrations $(p>0.300$ in all cases, see Table 1).

Effect of egg white AMPs concentrations on bacterial trans-shell infection (BTSI)

264 Concentrations of lysozyme and avidin did not affect incidence of BTSI $\left(\Delta\right.$ d.f. $=1, \chi^{2}=0.05, p$ $265=0.82$ and $\Delta$ d.f. $=1, \chi^{2}=0.01, p=0.999$, respectively; Table 2 ) and at the same time, incidence of BTSI was not affected by interaction between AMPs concentrations and experimental treatments ( $p>0.2$ in all cases; Table 2). There was also no correlation between intensities of BTSI and egg white lysozyme $\left(\Delta\right.$ d.f. $=1, \chi^{2}=0.42, p=0.517$; Table 2$)$ or avidin $\left(\Delta\right.$ d.f. $=1, \chi^{2}$ $=2.64, \mathrm{p}=0.104$; Table 2 ) concentrations in a subset of infected eggs.

\section{Effect of egg white AMPs concentrations on hatching success}

272 In our previous study, hatching success increased in partially incubated eggs ${ }^{13}$, yet here we demonstrated that it was not affected by lysozyme or avidin concentrations $\left(\Delta\right.$ d.f. $=1, \chi^{2}=1.26$, $\mathrm{p}=0.262$ and $\Delta$ d.f. $=1, \chi^{2}=1.58, \mathrm{p}=0.209$, respectively; Table 3). Similarly, we found no 
hatching success $(\mathrm{p}>0.1$ in all cases, Table 3$)$.

\section{Effect of egg white AMPs concentrations on hatchling phenotype}

279 Residual body mass and scaled BMIs of hatchlings were decreased in partially incubated eggs

$280 \quad\left(F_{(1,25)}=23.98, p<0.001\right.$ and $F_{(1,24)}=10.97, p=0.002$, respectively; Table 4). Accounting for 281 this source of variation, there was no effect of AMPs concentrations on residual body mass $282\left(\mathrm{~F}_{(1,24)}=0.02, \mathrm{p}=0.903\right.$ for lysozyme and $\mathrm{F}_{(1,24)}=0.01, \mathrm{p}=0.935$ for avidin; Table 4$)$. At the 283 same time, avidin failed to predict variation in scaled $\mathrm{BMI}\left(\mathrm{F}_{(1,23)}=0.14, \mathrm{p}=0.711\right.$; Table 4$)$. 284 However, scaled BMI decreased with increasing lysozyme concentration $\left(\mathrm{F}_{(1,24)}=7.23, \mathrm{p}=\right.$ 0.013) after statistical control for the variation induced by partial incubation (Table 4 and Figure 2).

\section{Discussion}

289 We found partial incubation affected concentrations of egg white AMPs in our study. Particularly, lysozyme concentration significantly increased and avidin shown only nonsignificant trend to increase in partially incubated eggs. In our previous experimental study, we have shown non-significant effect of partial incubation on changes in egg white lysozyme 293 concentration in quail and pigeon $\operatorname{eggs}^{30}$. On the other side, previous studies documented 294 decrease in egg white lysozyme concentration owing to full incubation in precocial chicken eggs $^{72}$ or in altricial red-capped lark eggs ${ }^{45}$, or slight decrease of egg white lysozyme in chicken eggs in the early phase of full incubation ${ }^{29,44}$. Based on these facts, it is apparent that 297 inconsistency exists in temperature-induced changes of egg white AMPs under different 298 incubation modes when various embryonic developmental stages might play a role ${ }^{37}$. In any 299 case, previous studies documented that lysozyme decrease in egg white owing to incubation is 300 a result of proteins aggregation ${ }^{42,43}$, binding of lysozyme to other proteins ${ }^{73}$, or lysozyme 
degradation early after incubation ${ }^{29}$. Yet, most recent works revealed that thermal aggregation

302 of proteins and resulted changes in protein abundances were highly dependent on the content 303 of particular amino acids such as arginine, lysine or aspartic acid acting as protein stabilizer 304 and/or destabilizers ${ }^{74,75}$. Moreover, temperature-induced level of proteins aggregation was 305 found to be linked with the concentrations of other heat-sensitive egg white proteins such as 306 ovotransferrin ${ }^{76}$. As proteomic and amino acids profile including arginine and ovotransferrin 307 significantly vary among species ${ }^{32,65}$, it is highly probable that increase of egg white lysozyme 308 owing to partial incubation in in mallard eggs might be related to various ratio and 309 concentrations of aggregation preventing arginine and/or ovotransferrin. In our study, we were 310 failed to analyse egg white ovotransferrin concentration due to high inconsistency in replicate 311 measurements and thus to test these associations. Yet, although above mentioned relationships 312 with aggregation preventing egg white substances require further experimental testing, it is 313 necessary to take them into account in future research focused on thermal properties of egg 314 white in birds. In our study, we did not find support for the role of egg white AMPs concentrations 316 preventing risk of bacterial trans-shell infection in vivo. Even if we revealed increased egg white 317 lysozyme in partially incubated eggs, neither lysozyme nor avidin egg white concentrations 318 affected incidence or intensity of bacterial trans-shell infection. These outcomes however do not explicitly imply costs for studied species or birds in general as in our previous experimental 320 study, egg whites enriched in ovo with hen egg white lysozyme significantly increased in vitro 321 antimicrobial action against indicator strains ${ }^{30}$. On the other side, there is no study evaluating 322 in vivo antimicrobial potential of naturally varied egg white AMPs and investigating associated microbial trans-shell infection. Besides, we have found in our previous study selective in vitro 324 antimicrobial activity of egg whites originated from precocial eggs treated with partial 325 incubation enhancing proliferation of a beneficial probiotic bacterial strain ${ }^{30}$. Similarly, 
incubation was documented to shift diversity of the eggshell microbiota from initial highly

327 diverse communities including opportunistic pathogens toward less diverse communities with

328 dominance of less harmful or even beneficial microorganisms ${ }^{6,77}$. It seems therefore that partial

329 incubation might be the mechanisms acting outside the eggs to modulate eggshell microbial

330 communities toward harmless or beneficial microorganisms and inside to maintain beneficial

331 bacterial invaders. Moreover, coupled protective role of incubation with egg white AMPs

332 against pathogenic microorganisms seems to be most effective only in early phase of embryonic

333 development, while developing extraembryonic structures seem to play a crucial protective role

334 later during incubation ${ }^{37,78}$.

335 Furthermore, we documented increased egg white LSM compromised body condition

336 of hatchlings expressed as scaled BMI. This is in accordance with our previous study

337 documenting growth-regulating role of egg white lysozyme as experimental increase in egg

338 white lysozyme in precocial quail (Coturnix japonica) eggs resulted in reduced tarsus length of

339 hatchlings ${ }^{49}$. Mechanisms of action could be inherent in the fact that LSM was documented to

340 have growth-regulating role in development of embryonic cartilage and skeletal structures ${ }^{79}$

341 including inhibition of mouse bone collagenase activity which may significantly affect

342 development of particular skeletal elements ${ }^{80}$. Effect of egg white avidin concentrations on

343 hatchlings phenotype was not apparent in this study. Although we documented egg white avidin

344 to alter chicks' phenotype in quail ${ }^{50}$, growth-inhibition effect of egg white avidin on chicks was

345 documented to be strongly dependent on egg weight since only chicks originated from lighter

346 eggs enriched in ovo with avidin hatched with reduced tarsus length. It follows, that although

347 egg white AMPs may fulfil protective antimicrobial role for the embryo during early phase of

348 embryo development, their increase in egg white may significantly compromised embryo

349 growth and negatively affect resulted phenotype of hatchlings in precocial birds. 
As in the case of partial incubation, we were failed to find any effect of clutch covering

351 with feather nest lining on egg white AMPs and related incidence and intensity of bacterial 352 trans-shell infection. Although clutch covering with nest lining was found to provide insulation 353 of eggs against ambient temperatures ${ }^{51,52}$, it seems that it rather helps to maintain eggs in 354 optimal temperatures around physiological zero to sustain eggs viability and improved 355 hatchability and hatchlings growth performance ${ }^{81-83}$, than to change temperature conditions to 356 level leading to temperature-induced changes in egg white AMPs profile. Nevertheless, only 357 two studies have shown that nest material and feather nest-lining have strong antimicrobial 358 potential with ability to shift especially eggshell microbiota in hoopoe (Upupa epops) $)^{10,28}$. As 359 evidence for such antimicrobial action of feather nest-lining in other bird species is lacking and 360 we did not find any effect on bacterial trans-shell infection via alterations of egg white AMPs, 361 it is highly possible that nest lining feather in our study species may provide antimicrobial 362 action only on the eggshells without effect on egg white AMPs and their antimicrobial potential, 363 or its primary function is clutch insulation and/or clutch protection against visually oriented 364 predators $^{56}$.

To conclude, we have shown in our study that partial incubation, as behavioural 366 mechanisms documented to have various functions ranging from antipredator nest protection 367 to maintaining egg viability, has also function to alter concentrations of egg white 368 antimicrobials during pre-incubation phase. Furthermore, we found that even if concentrations 369 of egg white AMPs were not associated with reduced intensity and incidence of bacterial trans370 shell infection in mallard eggs in vivo, increased concentration of particular egg white AMPs 371 had growth regulating role during embryogenesis at least in our precocial model species.

\section{Data availability}

374 The datasets generated during and/or analyzed during the current study are available from 
the corresponding author on reasonable request.

\section{References}

3781 Calatayud, M., Koren, O. \& Collado, M. C. Maternal Microbiome and Metabolic

379 Health Program Microbiome Development and Health of the Offspring. Trends

$380 \quad$ Endocrin. Met. 30, 735-744 (2019).

3812 Chen, C. Y. et al. Maternal gut microbes shape the early-life assembly of gut

382 microbiota in passerine chicks via nests. Microbiome 8, 129 (2020).

3833 Voirol, L. R. P., Weinhold, A., Johnston, P. R., Fatouros, N. E. \& Hilker, M. Legacy of 384 a Butterfly's Parental Microbiome in Offspring Performance. Appl. Environ. Microb. $385 \quad \mathbf{8 6}, 12(2020)$.

3864 Osorio, J. S. Gut health, stress, and immunity in neonatal dairy calves: the host side of 387 host-pathogen interactions. J. Anim. Sci. Biotechno. 11, 105 (2020).

3885 Campos-Cerda, F. \& Bohannon, B. J. M. The Nidobiome: A Framework for 389 Understanding Microbiome Assembly in Neonates. Trends Ecol. Evol. 35, 573-582 $390 \quad$ (2020).

3916 Grizard, S., Dini-Andreote, F., Tieleman, B. I. \& Salles, J. F. Dynamics of bacterial and fungal communities associated with eggshells during incubation. Ecol Evol 4, 393 1140-1157 (2014).

3947 van Veelen, H. P. J., Salles, J. F. \& Tieleman, B. I. Microbiome assembly of avian 395 eggshells and their potential as transgenerational carriers of maternal microbiota. Isme $396 \quad$ J. 12, 1375-1388 (2018).

3978 Diaz-Lora, S. et al. Experimental old nest material predicts hoopoe Upupa epops $398 \quad$ eggshell and uropygial gland microbiota. J. Avian Biol. 50, 9 (2019).

3999 Martinez-Garcia, A. et al. Nest Bacterial Environment Affects Microbiome of Hoopoe 
Eggshells, but Not That of the Uropygial Secretion. PloS ONE 11, 15 (2016).

40110 Ruiz-Castellano, C., Tomas, G., Ruiz-Rodriguez, M., Martin-Galvez, D. \& Soler, J. J.

402 Nest Material Shapes Eggs Bacterial Environment. PloS ONE 11, 21 (2016).

40311 van Veelen, H. P. J., Salles, J. F. \& Tieleman, B. I. Multi-level comparisons of cloacal, 404 skin, feather and nest-associated microbiota suggest considerable influence of 405 horizontal acquisition on the microbiota assembly of sympatric woodlarks and $406 \quad$ skylarks. Microbiome 5, 156 (2017).

40712 Cook, M. I., Beissinger, S. R., Toranzos, G. A., Rodriguez, R. A. \& Arendt, W. J. 408 Trans-shell infection by pathogenic micro-organisms reduces the shelf life of non409 incubated bird's eggs: a constraint on the onset of incubation? P. R. Soc. B 270, 2233$410 \quad 2240(2003)$.

41113 Javurkova, V., Albrecht, T., Mrazek, J. \& Kreisinger, J. Effect of intermittent 412 incubation and clutch covering on the probability of bacterial trans-shell infection. Ibis $413 \quad \mathbf{1 5 6}, 374-386(2014)$.

41414 Wang, C., Pors, S. E., Olsen, R. H. \& Bojesen, A. M. Transmission and pathogenicity 415 of Gallibacterium anatis and Escherichia coil in embryonated eggs. Vet. Microbiol. $416 \quad 217,76-81(2018)$.

41715 Lee, S. I., Lee, H., Jablonski, P. G., Choe, J. C. \& Husby, M. Microbial abundance on 418 the eggs of a passerine bird and related fitness consequences between urban and rural 419 habitats. PloS ONE 12, 17 (2017).

42016 Peralta-Sanchez, J. et al. Bacterial density rather than diversity correlates with 421 hatching success across different avian species. FEMS Microbiol. Ecol. 94, 3 (2018).

42217 Wang, J. M., Firestone, M. K. \& Beissinger, S. R. Microbial and environmental effects 423 on avian egg viability: Do tropical mechanisms act in a temperate environment? $424 \quad$ Ecology 92, 1137-1145 (2011). 
42518 Cook, M. I., Beissinger, S. R., Toranzos, G. A. \& Arendt, W. J. Incubation reduces microbial growth on eggshells and the opportunity for trans-shell infection. Ecol. Lett. 8, 532-537 (2005).

19 Cook, M. I., Beissinger, S. R., Toranzos, G. A., Rodriguez, R. A. \& Arendt, W. J. Microbial infection affects egg viability and incubation behavior in a tropical passerine. Behav. Ecol. 16, 30-36 (2005).

$43120 \quad$ Ishikawa, S. et al. Photodynamic antimicrobial activity of avian eggshell pigments. Febs Lett. 584, 770-774 (2010).

43321 Martin-Vivaldi, M. et al. Special structures of hoopoe eggshells enhance the adhesion of symbiont-carrying uropygial secretion that increase hatching success. J. Anim. Ecol. 83, 1289-1301 (2014).

22 D'Alba, L. et al. What Does the Eggshell Cuticle Do? A Functional Comparison of Avian Eggshell Cuticles. Physiol. Biochem. Zool. 90, 588-599 (2017). physiological adaptations of the eggs of two Australian megapodes to their nesting strategies and their implications for extinct titanosaur dinosaurs. J. Microsc.-Oxford 267, 237-249 (2017). Antimicrobial properties of a nanostructured eggshell from a compost-nesting bird. $J$. Exp. Biol. 217, 1116-1121 (2014).

44525 Bain, M. M. et al. Enhancing the egg's natural defence against bacterial penetration by 446 increasing cuticle deposition. Anim. Genet. 44; 661-668 (2013).

44726 Gautron, J., Rehault-Godbert, S., Pascal, G., Nys, Y. \& Hincke, M. T. Ovocalyxin-36 448 and other LBP/BPI/PLUNC-like proteins as molecular actors of the mechanisms of the avian egg natural defences. Biochem. Soc. T. 39, 971-976 (2011). 
27 Wellman-Labadie, O., Picman, J. \& Hincke, M. T. Antimicrobial activity of cuticle and outer eggshell protein extracts from three species of domestic birds. Brit. Poultry Sci. 49, 133-143 (2008).

45328 Ruiz-Castellano, C., Ruiz-Rodriguez, M., Tomas, G. \& Jose Soler, J. Antimicrobial activity of nest-lining feathers is enhanced by breeding activity in avian nests. FEMS Microbiol. Ecol. 95, 5 (2019).

29 Fang, J. et al. Liquefaction of albumen during the early incubational stages of the avian embryo and its impact on the antimicrobial activity of albumen. J. Food Agri. Environ. 10, 423-427 (2012). affect selective antimicrobial properties of the egg interior: experimental evidence from eggs of precocial and altricial birds. J. Exp. Biol. 222, 6 (2019).

31 Mann, K. \& Mann, M. In-depth analysis of the chicken egg white proteome using an LTQ Orbitrap Velos. Proteome Sci. 9, 6 (2011). Egg Albumen from Domestic Chicken, Duck, Goose, Turkey, Quail and Pigeon. Proteomics 17, 12 (2017).

33 Wellman-Labadie, O., Picman, J. \& Hincke, M. T. Comparative antibacterial activity of avian egg white protein extracts. Brit. Poultry Sci. 49, 125-132 (2008). (eds Y. Nys, M. Bain, \& F. V. Immerseel) 330-350 (Woodhead Publishing Ltd, 2011).

47135 Ibrahim, H. R. Innate Antimicrobial Proteins and Peptides of Avian Egg in Eggs as 472 Functional Foods and Nutraceuticals for Human Health (ed. Wu, J.) Ch. 12, 211-222 (Royal Soc. Chem., 2019).

47436 Ibrahim, H. R., Matsuzaki, T. \& Aoki, T. Genetic evidence that antibacterial activity of 
lysozyme is independent of its catalytic function. Febs Lett. 506, 27-32 (2001).

47637 Guyot, N. et al. Characterization of egg white antibacterial properties during the first half of incubation: A comparative study between embryonated and unfertilized eggs. Poultry Sci. 95, 2956-2970 (2016).

38 Guyot, N. et al. Proteomic analysis of egg white heparin-binding proteins: towards the identification of natural antibacterial molecules. Sci. Reports 6, 27974 (2016).

39 Pierce, E. C. et al. Bacterial-fungal interactions revealed by genome-wide analysis of bacterial mutant fitness. Nat. Microbiol. 6, 87-102 (2021).

Board, R. G. \& Fuller, R. Non-specificantimigrobial defences of avian egg, embryo and neonate. Biol. Rev. Camb. Philos. 49, 15-49 (1974).

41 Wu, J. P. \& Acero-Lopez, A. Ovotransferrin: Structure, bioactivities, and preparation. Food Res. Int. 46, 480-487 (2012).

Qiu, N. et al. Proteomic analysis of egg white proteins during the early phase of embryonic development. J. of Proteomics 75, 1895-1905 (2012).

Liu, Y. J., Qiu, N. \& Ma, M. H. Comparative proteomic analysis of egg white proteins during the rapid embryonic growth period by combinatorial peptide ligand libraries. Poultry Sci. 94, 2495-2505 (2015).

44 Fang, J. et al. Changes in the antimicrobial potential of egg albumen during the early stages of incubation and its impact on the growth and virulence response of Salmonella Enteritidis. Ital. J. Anim. Sci. 11, 92-97 (2012). Bacterial Communities of Eggshells and Antimicrobial Activities in Eggs during

49846 Ruiz-De-Castaneda, R., Vela, A. I., Gonzalez-Braojos, S., Briones, V. \& Moreno, J. 499 Drying eggs to inhibit bacteria: Incubation during laying in a cavity nesting passerine. 
Behav. Process. 88, 142-148 (2011).

50147 Ruiz-de-Castaneda, R., Vela, A. I., Lobato, E., Briones, V. \& Moreno, J. Early onset of incubation nad eggshell bacterial loads in temperate-zone-cavity-nesting passerine. Condor 114, 203-211 (2012).

50448 Bollinger, P. B., Bollinger, E. K., Daniel, S. L., Gonser, R. A. \& Tuttle, E. M. Partial 505 incubation during egg laying reduces eggshell microbial loads in a temperate-breeding 506 passerine. J. Avian Biol. 49, 6 (2018).

50749 Javůrkova, V., Krkavcová, E., Kreisinger, J., Hyršl, P. \& Hyanková, L. Effects of 508 experimentally increased in ovo lysozyme on egg hatchability, chicks complement 509 activity, and phenotype in a precocial bird. J. Exp. Zool. Part A 323, 497-505 (2015).

$51050 \quad$ Krkavcová, E., Kreisinger, J., Hyanková, L., Hyršl, P. \& Javůrkova, V. The hidden 511 function of egg white antimicrobials: egg weight-dependent effects of avidin on avian 512 embryo survival and hatchling phenotype. Biol. Open 7, 9 (2018).

51351 Pinowski, J. et al. The thermal properties of some nests of the Eurasian Tree Sparrow 514 Passer montanus. J. Therm. Biol. 31, 573-581 (2006).

51552 Prokop, P. \& Trnka, A. Why do grebes cover their nests? Laboratory and field tests of 516 two alternative hypotheses. J. Ethol. 29, 17-22 (2011).

51753 Wang, J. M. \& Beissinger, S. R. Partial incubation in birds: its occurrence, function, $518 \quad$ quantification. Auk 128, 454-466 (2011).

51954 D'Alba, L., Oborn, A. \& Shawkey, M. D. Experimental evidence that keeping eggs dry 520 is a mechanism for the antimicrobial effects of avian incubation. Naturwissenschaften $52199,1089-1095(2010)$.

52255 Morosinotto, C., Thomson, R. L. \& Korpimaki, E. Plasticity in incubation behaviour under experimentally prolonged vulnerability to nest predation. Behaviour 150, 1767$1786(2013)$. 

untangling the role of crypsis and parental behaviour. Funct. Ecol. 22, 872-879 (2008). yellow-legged gull. Behav. Ecol. Sociobiol. 64, 845-855 (2010).

$52958 \quad$ Loos, E. R. \& Rohwer, F. C. Laying-stage nest attendance and onset of incubation in prairie nesting ducks. Auk 121, 587-599 (2004).

53159 Oliveira, G. D., dos Santos, V. M., Rodrigues, J. C. \& Nascimento, S. T. Effects of different egg turning frequencies on incubation efficiency parameters. Poultry Sci 99, $4417-4420$ (2020).

$53460 \quad$ Finkler, M. S., Van Orman, J. B. \& Sotherland, P. R. Experimental manipulation of egg quality in chickens: influence of albumen and yolk on the size and body composition of near-term embryos in a precocial bird. J. Comp. Physiol B 168, 17-24 (1998).

53861 Bonisoli-Alquati, A. et al. Effects of egg albumen removal on yellow-legged gull chick phenotype. Funct. Ecol. 21, 310-316 (2007).

54062 Stubblefield, W. A. \& Toll, P. A. Effects of incubation-temperature and warm-water misting on hatching success in artificially incubated mallard duck eggs. Environ. Toxicol. Chem. 12, 695-700 (1993).

54363 Sellier, N. et al. Comparison of fertility and embryo mortality following artificial 544 insemination of common duck females (Anas Platyrhynchos) with semen from common or Muscovy (Cairina Moschata) drakes. Theriogenology 64, 429-439 (2005).

54664 Osserman, E. F. \& Lawlor, D. P. Serum and urinary lysozyme (muramidase) in monocytic and monomyelocytic leukemia. J. Exp. Med. 124, 921-952 (1966).

54865 Shawkey, M. D. et al. Do birds differentially distribute antimicrobial proteins within 549 clutches of eggs? Behav. Ecol. 19, 920-927 (2008). 
55066 Horz, H. P., Vianna, M. E., Gomes, B. \& Conrads, G. Evaluation of universal probes

$551 \quad$ and primer sets for assessing total bacterial load in clinical samples: General

552 implications and practical use in endodontic antimicrobial therapy. J. Clin. Microbiol.

$553 \quad 43,5332-5337(2005)$.

55467 Peig, J. \& Green, A. J. New perspectives for estimating body condition from

555 mass/length data: the scaled mass index as an alternative method. Oikos 118, 1883-

$5561891(2009)$.

55768 R-Core-Team. R: A language and environment for statistical computing,

558 http://www.R-proje ct.org/ (2020).

55969 RStudio Team. RStudio: Integrated Development for R. RStudio, Inc., Boston, MA

$560 \quad$ URL http://www.rstudio.com/ (2015).

$56170 \quad$ Bates, D., Machler, M., Bolker, B. M. \& Walker, S. C. Fitting Linear Mixed-Effects

562 Models Using lme4. J. Sta. Softw. 67, 1-48 (2015).

56371 Crawley, M. J. The R Book (John Wiley \& Sons, Ltd, 2007).

56472 Cunningham, F. E. Changes in egg-white during incubation of fertile egg. Poultry Sci.

$565 \quad 53,1561-1565(1974)$.

56673 Kato, A., Imoto, T. \& Yagishita, K. Binding groups in ovomucin-lysozyme interaction.

567 Agri. Biol. Chem. Tokyo 39, 541-544 (1975).

56874 Hong, T. H., Iwashita, K., Handa, A. \& Shiraki, K. Arginine prevents thermal

569 aggregation of hen egg white proteins. Food Res. Int. 97, 272-279 (2017).

57075 Anumalla, B. \& Prabhu, N. P. Counteracting Effect of Charged Amino Acids Against

571 the Destabilization of Proteins by Arginine. Appl. Biochem. Biotech. 189, 541-555

$572 \quad$ (2019).

57376 Iwashita, K., Handa, A. \& Shiraki, K. Co-aggregation of ovotransferrin and lysozyme.

$574 \quad$ Food Hydrocolloid 89, 416-424 (2019). 
57577 Lee, W. Y., Kim, M., Jablonski, P. G., Choe, J. C. \& Lee, S. I. Effect of Incubation on 576 Bacterial Communities of Eggshells in a Temperate Bird, the Eurasian Magpie (Pica 577 pica). PloS ONE 9, 11 (2014).

57878 Hincke, M. T. et al. Dynamics of Structural Barriers and Innate Immune Components 579 during Incubation of the Avian Egg: Critical Interplay between Autonomous

580 Embryonic Development and Maternal Anticipation. J. Innate Imm 11, 111-124 $581 \quad$ (2019).

58279 Kuettner, K. E. et al. Lysozyme in epiphyseal cartilge. II. The effect of egg white 583 lysozyme on mouse embryonic femurs in organ cultures. J. Cell Biol. 44, 329-339 $584 \quad$ (1970).

$58580 \quad$ Sakamoto, S., Sakamoto, M., Goldhaber, P. \& Glimcher, M. J. In hibitionof mouse 586 bone collagenase by lysozyme. Calc. Tiss. Res. 14, 291-299 (1974).

$58781 \quad$ Peralta-Sanchez, J. M., Moller, A. P. \& Soler, J. J. Colour composition of nest lining 588 feathers affects hatching success of barn swallows, Hirundo rustica (Passeriformes: 589 Hirundinidae). Biol. J. Linn. Soc. 102, 67-74 (2011).

59082 Dawson, R. D., O'Brien, E. L. \& Mlynowski, T. J. The price of insulation: costs and 591 benefits of feather delivery to nests for male tree swallows Tachycineta bicolor. $J$. 592 Avian Biol. 42, 93-102 (2011).

59383 Stephenson, S., Hannon, S. \& Proctor, H. The function of feathers in tree swallow 594 nests: insulation or ectoparasite barrier? Condor 111, 479-487 (2009).

\section{Acknowledgements}

597 We thank to Eva Krkavcová for the lab works and AMPs concentrations analyses. This study 598 was supported by institutional research support of the Czech Academy of Sciences RVO: 59968081766. 
601 V.G.J. and J.K. conceptualized research and proposed experimental design. V.G.J. acquired 602 funding and managed the project. J.K. conducted statistics, graphical visualization and wrote 603 results. J.S and V.G.J. wrote the main manuscript text. All authors reviewed the manuscript.

604

605 Additional information

606 Competing interests

607 The authors declare no competing interests.

608

609

610

611

612

613

614

615

616

617

618

619

620

621

622

623

624 
625 Table 1. Egg white AMP concentration - A) Avidin and B) Lysozyme as a response of partial 626 incubation, clutch covering with feather nest lining and their interactions. Step-wise elimination 627 of non-significant terms was used to select the best minimal adequate model (MAM). Predictors 628 retained in the minimal adequate model (MAM) after step-wise elimination of nonsignificant 629 variables are in bold. Significance (p) was assessed based on deviance change $(\chi 2)$ and 630 corresponding degrees of freedom $(\Delta$ D.f. $)$

631

632

\begin{tabular}{llccc}
\hline \multirow{2}{*}{ Response } & \multicolumn{1}{c}{ Predictor } & $\Delta$ D.f. & $\chi^{2}$ & $\mathrm{p}$ \\
\hline \multirow{2}{*}{ A) Avidin } & Partial incubation & 1 & 3.281 & 0.070 \\
& Clutch covering & 1 & 0.000 & 0.987 \\
& Partial incubation x Clutch covering & 1 & 0.005 & 0.946 \\
\hline \multirow{2}{*}{ B) Lysozyme } & Partial Incubation & $\mathbf{1}$ & $\mathbf{2 5 . 7 1 6}$ & $<\mathbf{0 . 0 0 1}$ \\
& Clutch covering & 1 & 0.755 & 0.385 \\
& Partial incubation x Clutch covering & 1 & 0.951 & 0.330
\end{tabular}

633

634

635

636

637

638

639

640

641

642

643

644 
645 Table 2. Variation in bacterial trans-shell infection (BTSI) - A) prevalence and B) intensity due 646 to the effect of egg white AMPs concentrations, partial incubation, clutch covering, and their 647 interactions. Predictors retained in the minimal adequate model after step-wise elimination of 648 nonsignificant variables are bold. Shown are probability values (p), values of $\chi 2$ statistics and 649 associated degrees of freedom

650

\begin{tabular}{llccc}
\hline Response & \multicolumn{1}{c}{ Predictor } & $\Delta$ D.f. & $\chi^{2}$ & $p$ \\
\hline A) BTSI Prevalence & Lysozyme & 1 & 1.619 & 0.203 \\
& Avidine & 1 & 0.001 & 0.981 \\
& Partial incubation & 1 & 0.052 & 0.820 \\
& Clutch covering & 1 & 0.052 & 0.820 \\
& Lysozyme x Partial incubation & 1 & 0.209 & 0.648 \\
& Lysozyme x Clutch covering & 1 & 0.488 & 0.485 \\
& Avidin x Partial incubation & 1 & 0.660 & 0.417 \\
& Avidin x Clutch covering & 1 & 0.328 & 0.567 \\
B) BTSI Intensity & Incubation & 1 & 3.071 & 0.080 \\
& Avidin & 1 & 2.642 & 0.104 \\
& Lysozyme & 1 & 0.420 & 0.517 \\
& Clutch covering & 1 & 0.420 & 0.517 \\
& Lysozyme x Partial incubation & 1 & 1.480 & 0.224 \\
& Lysozyme x Clutch covering & 1 & 0.772 & 0.379 \\
& Avidin x Partial incubation & 1 & 0.037 & 0.847 \\
& Avidin x Clutch covering & 1 & 0.305 & 0.581
\end{tabular}


661 Table 3. Variation in hatching success due to the effect of egg white AMPs concentrations, 662 partial incubation, clutch covering with feather nest lining and their interactions. Predictors 663 retained in the minimal adequate model (MAM) after step-wise elimination of nonsignificant 664 variables are in bold. Shown are probability values (p), values of $\chi^{2}$ statistics and associated 665 degrees of freedom

666

\begin{tabular}{lccc}
\hline \multicolumn{1}{c}{ Predictor } & $\Delta$ D.f. & $\chi^{2}$ & $\mathrm{p}$ \\
\hline Partial incubation & $\mathbf{1}$ & $\mathbf{8 . 7 9 6}$ & $\mathbf{0 . 0 0 3}$ \\
Lysozyme & 1 & 1.257 & 0.262 \\
Avidin & 1 & 1.575 & 0.209 \\
Clutch covering & 1 & 1.575 & 0.209 \\
Lysozyme x Partial incubation & 1 & 0.645 & 0.422 \\
Lysozyme x Clutch covering & 1 & 2.579 & 0.108 \\
Avidin x Partial incubation & 1 & 0.080 & 0.778 \\
Avidin x Clutch covering & 1 & 0.844 & 0.358
\end{tabular}

668

669

670

671

672

673

674

675

676

677

678

679 
Table 4. Variation in morphometric parameters - A) body mass adjusted for egg volume and B)

681 scaled body mass index (BMI) due to the effect of egg white AMPs concentrations and partial 682 incubation. Data were analyzes using GLMM assuming Gaussian distribution of residuals.

683 Predictors retained in the minimal adequate model after step-wise elimination of nonsignificant 684 variables are in bold. Shown are probability values (p), values of $F$ statistics and associated 685 degrees of freedom

686

\begin{tabular}{llccc|}
\hline Response & \multicolumn{1}{c}{ Predictor } & D.f. & F & p \\
\hline A) residual body mass & Partial incubation & $(1,26)$ & $\mathbf{2 3 . 9 8 2}$ & $\mathbf{0 . 0 0 0}$ \\
& Lysozyme & $(1,25)$ & 0.015 & 0.903 \\
& Avidin & $(1,24)$ & 0.007 & 0.935 \\
\hline \multirow{2}{*}{ B) scaled BMI } & Lysozyme & $(1,25)$ & $\mathbf{1 0 . 9 6 5}$ & $\mathbf{0 . 0 0 3}$ \\
& Partial incubation & $(1,25)$ & $\mathbf{7 . 2 2 7}$ & $\mathbf{0 . 0 1 3}$ \\
& Avidin & $(1,24)$ & 0.140 & 0.711
\end{tabular}

687

688

689

690

691

692

693

694

695

696

697

698

699 
700 Figure legends:

701 Figure 1. Variation of A) lysozyme and B) avidin concentrations in mallard eggs treated with 702 partial incubation (incub.) vs. control un-incubated eggs (unincub.). GLMM-based probability 703 values are shown

704

705 Figure 2. Effect of lysozyme concentrations on scaled BMI of mallard hatchlings $(\mathrm{n}=27)$.

706 Regression were adjusted for the effect of partial incubation treatment. Predictions and 95\% 707 confidence intervals are shown

708

709 
Figures



\section{B) Avidin}

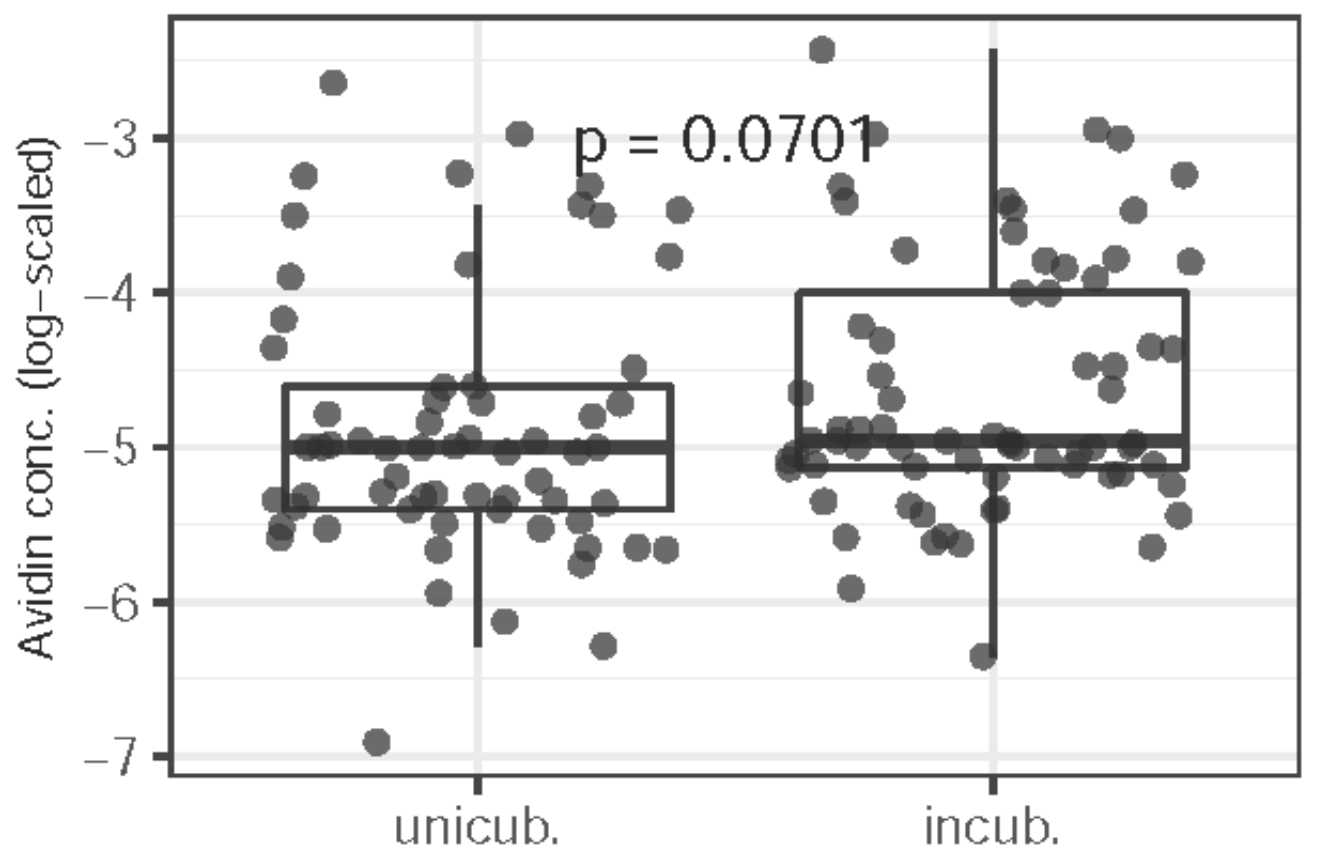

Figure 1

Variation of A) lysozyme and B) avidin concentrations in mallard eggs treated with partial incubation (incub.) vs. control un-incubated eggs (unincub.). GLMM-based probability values are shown 


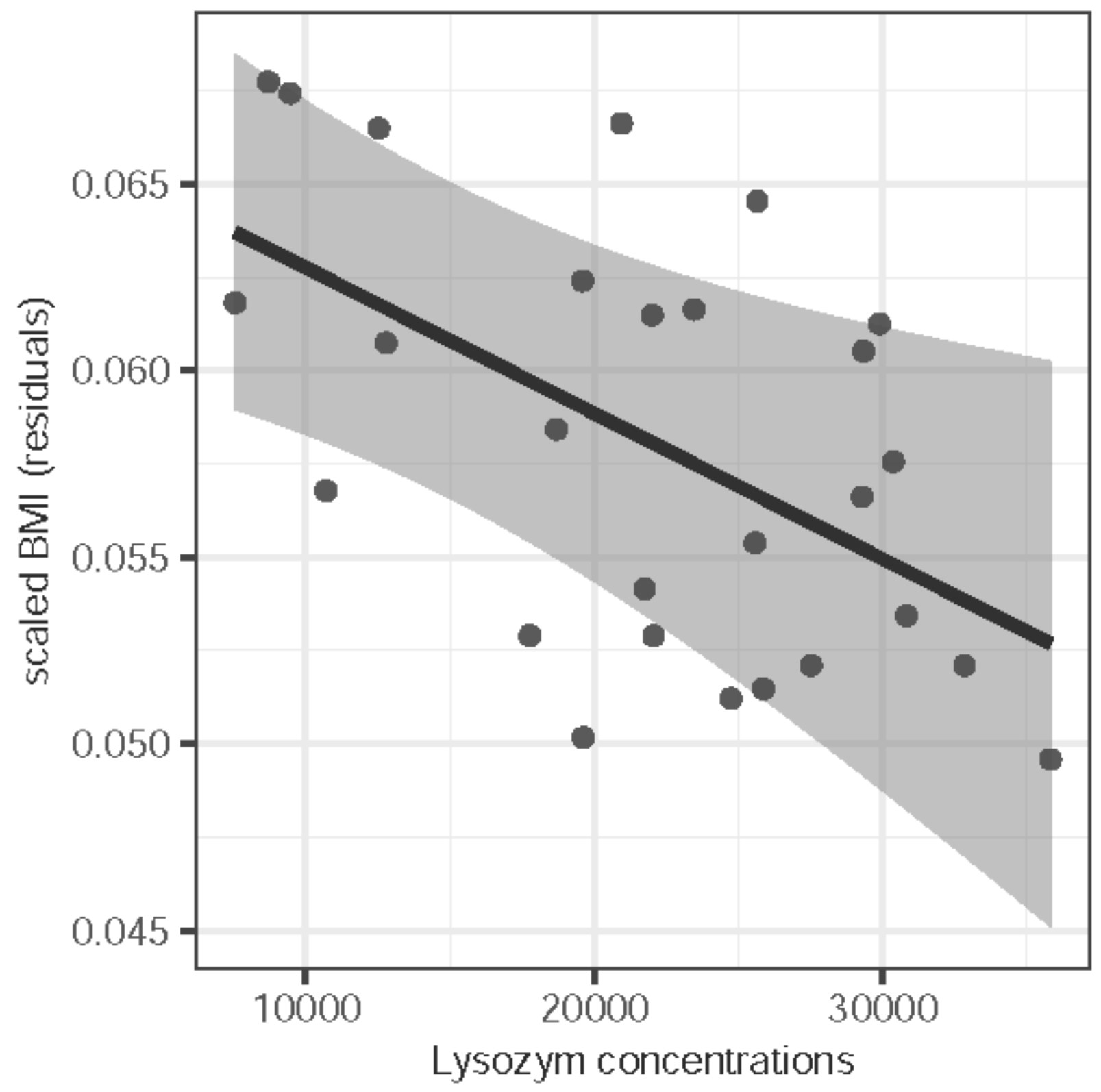

Figure 2

Effect of lysozyme concentrations on scaled BMI of mallard hatchlings $(n=27)$. Regression were adjusted for the effect of partial incubation treatment. Predictions and $95 \%$ confidence intervals are shown 